P70 CAN ENDOBRONCHIAL ULTRASOUND (EBUS) GUIDED TRANSBRONCHIAL NEEDLE ASPIRATION (TBNA) BE USED AS A FIRST LINE INVESTIGATION IN THE DIAGNOSIS OF CENTRAL LUNG PARENCHYMAL LESIONS?

doi:10.1136/thoraxjnl-2012-202678.312

S Leyakathali Khan, M Haris, S Diver, B Miller, M Munavvar. Royal Preston Hospital, Preston, UK

Background Accurate diagnosis and staging remains a cornerstone in the management of patients with lung cancer. EBUS-TBNA has been demonstrated to be a safe and minimally invasive tool to evaluate mediastinal and hilar lymph nodes in patients with suspected lung cancer. It has also shown to be effective in sampling central lung parenchymal lesions with no obvious bronchoscopic findings. The aim of the study was to evaluate the role of EBUS TBNA as an early investigative tool in the diagnosis of lung cancer with central lung parenchymal lesions.

Methods Retrospective study of 119 consecutive patients who had EBUS-TBNA from paratracheal and peribronchial masses between January 2009 and February 2012. All patients had a nondiagnostic flexible bronchoscopy just before having EBUS. 15 of these also had mediastinal or hilar lymph node sampling at the time of procedure.

Results $N=119$, mean age 68 years (SD 10); male: female, $56(\%)$ : $63(\%) .37$ paratracheal and 82 peribronchial lesions were identified using convex-probe EBUS. 5 with no further data were excluded.

Of the 114 samples, 110 were diagnostic confirming lung malignancy $107(97 \%)$, lymphoma $1(1 \%)$ and bronchial cysts $2(2 \%)$; 1 sample was insufficient and 3 showed no malignant cells (1 had surgical biopsy confirming squamous cell carcinoma, 1 was benign on follow imaging, 1 had CT guided biopsy positive for adenocarcinoma, 1 was not suitable for further invasive tests but CT showed progressive changes suggestive of lung malignancy).

Of the 107lung malignancy, 31 wereadenocarcinoma, 34 squamous cell carcinoma, 25 small cell carcinoma, 12 non-small cell carcinomanot otherwise specified (NOS), 3 malignant cells - NOS and 2 extrapulmonary metastases. The sensitivity of EBUS-TBNA for the diagnosis of lung cancer was 97\% (95\% confidence interval $92-99 \%$ ) with a negative predictive value $56 \%$ (95\% CI 26-83\%).

Conclusion Our results show that EBUS TBNA has a very high sensitivity in the diagnosis of central lung masses related to lung cancer. It could be used as a "one stop" as an early minimally invasive tool in the lung cancer diagnostic pathway to enable accurate diagnosis and a negative result may warrant other invasive tests.

\section{P71 PATIENT SATISFACTION DURING ENDOBRONCHIAL ULTRASOUND-GUIDED TRANSBRONCHIAL NEEDLE ASPIRATION PERFORMED UNDER MILD CONSCIOUS SEDATION}

doi:10.1136/thoraxjnl-2012-202678.313

A Jeyabalan, H Lockman. ARL Medford North Bristol Lung Centre, Southmead Hospital, Bristol, UK

Introduction Endobronchial ultrasound-guided transbronchial needle aspiration (EBUS-TBNA) is an increasingly used mediastinal sampling technique recommended by $\mathrm{NICE}^{1}$ for staging and diagnosis of non-small cell lung cancer. It is also used to diagnose unexplained accessible mediastinal adenopathy and lesions. Many EBUS-TBNA centres use conscious sedation in an ambulatory setting to optimise patient flow, save costs and shorten recovery time. The size of the EBUS-TBN. A scope with an external diameter of just below $7 \mathrm{~mm}$ requires it to be inserted orally, which has been associated with lower patient satisfaction at bronchoscopy in the past. ${ }^{2}$ There is only one published EBUS-TBN. A patient satisfaction study which used deep conscious sedation with propofol and was not a UK study. ${ }^{3}$ This study was carried out to investigate the experience of patients undergoing EBUS-TBNA using mild conscious sedation without propofol in a UK setting.

Methods 45 consecutive patients requiring EBUS-TBNA for investigation of unexplained mediastinal adenopathy were invited to complete a questionnaire post procedure. EBUS-TBNA was performed under light conscious sedation as previously described. ${ }^{4}$ The average dose of intravenous sedatives was also recorded.

Results The average age of the $38 / 45$ (84\%) patients who completed the questionnaire was 59.0 years and 24/33 (72\%) patients were male. Five patients had previously undergone fibreoptic bronchoscopy. The most common reason for referral was for diagnosis of suspected malignancy (30/45, 67\%).

All patients felt that they had been provided with adequate written and verbal information, been given adequate opportunity to ask questions and had all potential risks explained to them prior to the test.

The average dose of sedative agents administered was $59.4 \mathrm{mcg}$ fentanyl and $3.2 \mathrm{mg}$ midazolam. The most commonly reported symptom was cough in 35 (92\%) patients. Of these patients, 27 $(78 \%)$ described the severity as being mild. All but 3 patients $(35 / 38$, $92 \%)$ stated that they would definitely or probably undergo a repeat EBUS-TBNA. No complications were reported.

Conclusions This UK single centre study demonstrates that EBUS-TBNA under mild conscious sedation (without propofol) is a well tolerated procedure with patients reporting a high degree of satisfaction with both the test and the information received prior to having the test.

\section{References}

1. The diagnosis and Treatment of Lung Cancer. NICE clinical guideline. http://publications.nice.org.uk/lung-cancercg121. Accessed 11th June 2012

2. Lechtzin N, Rubin HR, White $P$ et al. Patient satisfaction with bronchoscopy. Am J Resp Crit Care Med 2002; 166(10): 1326-31.

3. Steinfort DP, Irving LB. Patient satisfaction during endobronchial ultrasound-guided transbronchial needle aspiration performed under conscious sedation. Respiratory Care 2010; 55(6): 702-6.

4. Medford AR, Agrawal S, Free CM et al. A performance and theoretical cost analysis of endobronchial ultrasound-guided transbronchial needle aspiration in a UK tertiary respiratory centre. QJM 2009; 102(12): 859-64.

\section{P72 THE USE OF ENDOSCOPIC ULTRASOUND IN THE DIAGNOSIS OF MEDIASTINAL LYMPHADENOPATHY: A COMPARISON OF TRUCUT BIOPSY VERSUS FINE NEEDLE ASPIRATION}

doi:10.1136/thoraxjnl-2012-202678.314

P Ramachandran, F Al-Murani, R Banka King. George Hospital, Ilford, UK

Background Endoscopic ultrasound guided fine needle aspiration is a commonly used tool in the diagnosis of mediastinal lymphadenopathy. However, less is known of the value of additional trucut biopsy. This retrospective study compares the outcomes of EUSguided FNA and trucut biopsy, in an unselected group of patients with mediastinal lymphadenopathy of unknown origin.

Method The endoscopy database of two district general hospital sites was reviewed for all patients who had undergone endoscopic ultrasound for mediastinal lymphadenopathy. Results were reviewed for all patients who had cytological or histological specimens taken. Documentation of patient comfort levels and immediate complications was also collected.

Results 77 patients underwent EUS for mediastinal lymphadenopathy and had specimens taken via FNA, trucut or both. For 1 patient 